\title{
Characterization of Human Osteoarthritic Cartilage Using Optical and Magnetic Resonance Imaging
}

\author{
Ella F. Jones, Joseph Schooler, David C. Miller, Christopher R. Drake, Hilla Wahnishe, \\ Sarmad Siddiqui, Xiaojuan Li, Sharmila Majumdar
}

Department of Radiology and Biomedical Imaging, Center for Molecular and Functional Imaging, University of California, 185 Berry Street, Suite 350, Box 0946, San Francisco, CA 94107, USA

\begin{abstract}
Purpose: Osteoarthritis $(\mathrm{OA})$ is a degenerative disease starting with key molecular events that ultimately lead to the breakdown of the cartilage. The purpose of this study is to use two imaging methods that are sensitive to molecular and macromolecular changes in OA to better characterize the disease process in human osteoarthritic cartilage.

Procedures: Human femoral condyles were collected from patients diagnosed with severe OA during total knee replacement surgeries. $T_{1 \rho}$ and $T_{2}$ magnetic resonance measurements were obtained using a 3-Tesla whole body scanner to assess macromolecular changes in the damaged cartilage matrix. Optical imaging was performed on specimens treated with MMPSense 680 to assess the matrix metalloproteinase (MMP) activity. A linear regression model was used to assess the correlation of MMP optical data with $T_{1 \rho}$ magnetic resonance (MR) measurements. Slices from a representative specimen were removed from regions with high and low optical signals for subsequent histological analysis.

Results: All specimens exhibit high $T_{1 \rho}$ and $T_{2}$ measurements in the range of 48-75 ms and 36$69 \mathrm{~ms}$, respectively. They also show intense photon signals $\left(0.376\right.$ to $\left.7.89 \times 10^{-4} \mathrm{~cm}^{2}\right)$ from the activated MMPSense 680 probe, indicative of high MMP activity. The analysis of variance test of the regression model indicates a positive correlation between the MMP optical signal and $T_{1 \rho}$ measurements $\left(R^{2}=0.8936, P=0.0044\right)$. Histological data also confirmed that regions with high MMP optical signal and intense $T_{1 \rho}$ relaxation exhibit severe clefting, abnormal tidemarks, and irregular cellularity.

Conclusions: The high $T_{1 \rho}$ and $T_{2}$ measurements suggest that there is a severe loss of proteoglycans with high water mobility in the damaged cartilage. The intense optical signals found in these specimens indicate the presence of active MMPs, and the positive correlation with $T_{1 \rho}$ measurements implicates MMP's involvement in OA progression, characterized by a severe loss of proteoglycans in the cartilage matrix. The bimodal approach using optical and MR imaging may provide key molecular and macromolecular information of the disease pathway, offering insights toward the development of new tools for the early detection, treatment, and/or prevention of OA.
\end{abstract}

Key words: Osteoarthritis, Optical imaging, MR imaging, Proteoglycans, Matrix metalloproteinase 
$\mathrm{O}$ steoarthritis (OA) is a degenerative joint disease that affects more than 27 million people in the U.S., costing approximately $\$ 34$ billion in health care every year [1]. OA is characterized by degeneration of cartilage in joints. In more advanced cases, total loss of the cartilage between joints may cause physical disability. OA is more prevalent in the aging population, and by the age of 70 , approximately $70 \%$ of the population will show radiological evidence of the disease [2]. However, younger people with obesity, occupational stress, or people who have suffered a traumatic injury are also at risk. Because there is no effective disease modifying therapy for $\mathrm{OA}$, it is critical to develop tools for the detection and characterization of the disease in its early stages, so that the knowledge gained can be used to devise new regimens to slow down, reverse, or prevent the process of cartilage degeneration.

Magnetic resonance (MR) imaging of cartilage has been used widely as a tool for diagnosing joint pathology. It offers multiplanar image acquisition with high spatial resolution, giving superior depiction of soft tissue details. Standard MR imaging is used to assess cartilage damage by examining the reduction in cartilage thickness or volume. In $\mathrm{OA}$, these morphological changes are often observed when the disease has already advanced to its late stages. In the past, several more complex MR techniques have been developed to detect and quantify macromolecular changes in OA [3-5]. For example, $T_{2}$ relaxation mapping (the spinspin relaxation time) predominantly measures the changes in the collagen component and hydration in the extracellular matrix $[6,7]$. More recently, $T_{1 \rho}$ relaxation mapping (the spin-lattice relaxation time in the rotating frame) has been correlated with the loss of proteoglycan (PG) macromolecules that occurs during the early stages of OA in bovine [810] and human cartilages [11]. Both $T_{2}[12,13]$ and $T_{1 \rho}[14$, 15] mapping techniques have been shown to be able to detect early cartilage degeneration in vivo. These techniques have shown great promises for the direct and indirect measurement of macromolecular changes in early cartilage failure. However, the earliest pathological signaling pathway that causes macromolecular changes and ultimately structural-functional degradation is unclear.

Inflammation is recognized as a major contributing process to both the symptoms and progression of OA [16]. It starts at the molecular level with the activation of key signaling pathways which results in the breakdown of the cartilage framework that ultimately leads to irreversible degeneration. Hence, these signaling pathways are critical to the onset and progression of the disease. Collective evidence has indicated that matrix metalloproteinases (MMPs), a family of zinc-dependent proteolytic enzymes, are involved in arthritic diseases [17, 18]. Among the family of 16 secreted and seven membrane-tethered human MMPs, the gelatinase (MMP-2 and -9) and collagenase (MMP-1 and -13) subclasses are of particular importance owing to their ability to digest collagen, basement membranes, and other macromolecules in the extracellular matrix $[19,20]$. These enzymes are produced as inactive precursors and can subsequently be activated proteolytically by active MMPs upon secretion into the extracellular space [21]. In vitro studies of macrophages and chondrocytes have provided a more comprehensive molecular mechanism of pro-MMP production and activation, as well as their concerted involvement in OA degeneration [22]. Based on this signaling pathway, the upregulation of MMP-9 and -13 are considered as potential targets for early assessment and therapeutic intervention for OA.

In the past decade, there has been a rapid growth of molecularly targeted imaging agents for disease detection [23]. MMPs have been identified as imaging targets for inflammation [24], cardiovascular disease [25], and cancer [26]. The first MMP activatable probe for near-IR (NIR) optical imaging was described by Weissleder et al. [27]. This "smart" probe comprises a PEGylated poly-(L-lysine) backbone, self-quenching NIR fluorophores and a peptide sequence (GPLGVRGKC) susceptible to MMP proteolytic activities [28]. In the absence of MMPs, the self-quenched probe is optically silent. Upon proteolytic cleavage, the peptidic substrate fragments containing the fluorophores are released from the backbone, and the probe is "turned on" resulting in significant amplification of the fluorescent signal at $695 \mathrm{~nm}$. Owing to this activation mechanism, the MMP probe is regarded as being highly specific and sensitive for MMP activity in NIR optical imaging.

Although the MMP probe was originally designed to target MMP-2, further development and optimization by the commercial supplier (it is now called MMPSense, Perkin Elmer, Boston, MA) has shown that it is predominantly activated by MMP-9 and -13 , with lower activation by a number of MMPs including MMP-2, -7, and -12 [29] [30] (Fig. 1). While MMPSense continues to demonstrate its ability to detect and define MMP activities in the tumor microenvironment [31-33] and rheumatoid arthritis [34], the utility of this probe for the characterization of OA is under explored. Herein, we present our effort to combine optical

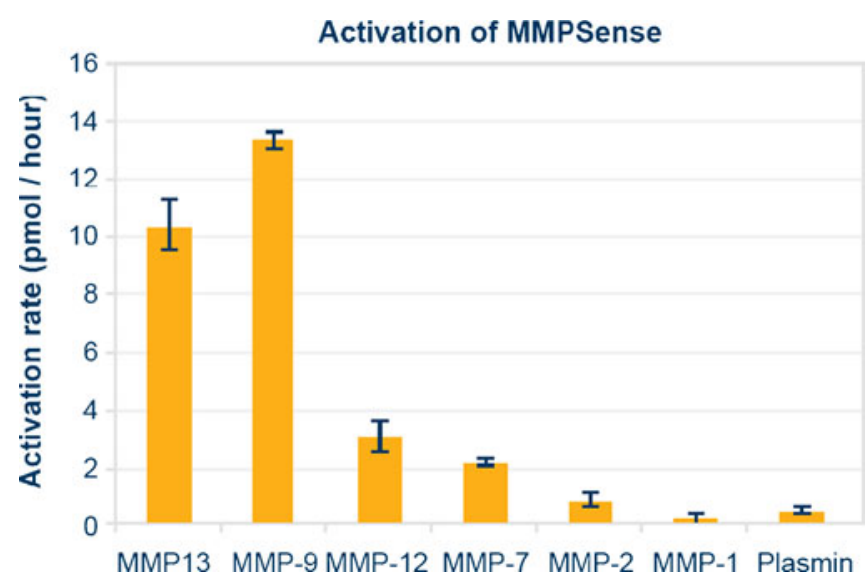

Fig. 1. The overall proteolytic activity of MMPSense in an enzyme panel showing this probe is predominantly activated by MMP-9 and -13. Courtesy of VisEn Medical, Inc. 
and MR imaging techniques to characterize both MMP activity and proteoglycan loss in an attempt to provide a more comprehensive profile of the degradation process in human osteoarthritic cartilage.

\section{Materials and Methods}

\section{Human Knee Specimen Preparation}

Human femoral condyles were collected from patients diagnosed with severe OA during total knee replacement surgeries at the UCSF Arthritis and Joint Replacement Center. All specimens $(n=6)$ were preserved immediately in dry ice, then stored in a $-80^{\circ} \mathrm{C}$ freezer no later than $3 \mathrm{~h}$ post surgery. Prior to imaging experiments, the specimens were wrapped in gauzes soaked with Lactated Ringer's solution (Braun Medical Sciences, Irvine, CA) to curtail articular cartilage dehydration and stored in a $4{ }^{\circ} \mathrm{C}$ refrigerator for $2-4 \mathrm{~h}$ to thaw.

\section{Ex Vivo MR Imaging}

Upon thawing of the specimen, a layer of simithecone (Spectrum Chemical Manufacturing, Gardena, CA) was applied to the articular surface of the condyle to reduce biochemical exchanges between the specimen and surrounding solution and to minimize the formation of air bubbles. The specimen was then attached to a plastic grid for spatial reference. The specimen and grid were fixated into a cylindrical plastic container and immersed in phosphate-buffered saline (PBS). The total preparation time before MR imaging was no more than $30 \mathrm{~min}$.

MR imaging was performed on a 3-Tesla whole body scanner (Signa HDx, GE Healthcare, Milwaukee, WI) using an eightchannel phased array knee coil (Invivo, Orlando, FL). All specimens were scanned in their respective physiological positions to simulate their in vivo orientation and position. The sequences used were (1) $T_{1}$-weighted three-dimensional high spatial resolution volumetric fat-suppressed spoiled gradient echo (SPGR) sequence $\left(\mathrm{TR} / \mathrm{TE}=20 / 7.5 \mathrm{~ms}, 12^{\circ}\right.$ flip angle, $512 \times 512$ matrix, $0.293 \times 0.293$ $\mathrm{mm}$ in-plane spatial resolution, $16-\mathrm{cm}$ field of view, 1-mm section thickness, number of slices $=40$; acquisition time of $7 \mathrm{~min} 37 \mathrm{~s}$ ); (2) three-dimensional $T_{1 \rho}$ mapping sequence consisted of magnetization preparation for imparting $T_{1 \rho}$ contrast and 3-D SPGR acquisition immediately after the $T_{1 \rho}$ preparation during transient signal evolution [35]. The main parameters used for this sequence were as follows: TR/TE=9.3/3.7 ms; 14-cm field of view; $256 \times 192$ matrix; 2-mm section thickness; number of slices $=20$; receiver bandwidth of $31.25 \mathrm{kHz}$; 48 views per segment; recovery time of $1.5 \mathrm{~s}$; spin-lock times of $0,10,40$, and $80 \mathrm{~ms}$; and spin-lock frequency of $500 \mathrm{~Hz}$. The total acquisition time was $12 \min 42 \mathrm{~s}$; (3) Sagittal 3-D $T_{2}$ maps were acquired by adding a nonselective $T_{2}$ preparation imaging sequence to the SPGR sequence [36] using echo times of $4.1,14.5,25.0$, and $45.9 \mathrm{~ms}$. All other prescription parameters for the $T_{2}$ sequence were identical to those used for the $T_{1 \rho}$ sequence, and the total acquisition time was $10 \min 36 \mathrm{~s}$.

\section{Ex Vivo Image Processing}

After scanning, all MR images were transferred to a computer workstation (Sun Microsystems, Palo Alto, CA) for offline post- processing. The $T_{1 \rho}$ and $T_{2}$ maps were reconstructed using an in-house developed software written in $\mathrm{C}$ by fitting the $T_{1 \rho^{-}}$ weighted and $T_{2}$-weighted images pixel by pixel to the equations

$$
S(\mathrm{TSL}) \propto S_{0} \times e^{\left(\frac{\mathrm{TSL}}{T_{1_{\rho}}}\right)} ; S(\mathrm{TE}) \propto S_{0} \times e^{\left(\frac{\mathrm{TE}}{T_{2}}\right)},
$$

respectively, whereas TSL stands for time of spin-lock and TE stands for echo time, $S_{0}$ is the signal intensity without $T_{1 \rho}$ or $T_{2}$ weight, and $S$ is signal intensity with $T_{1 \rho}$ or $T_{2}$ weight.

Cartilage was segmented semi-automatically in sagittal SPGR images using an in-house developed program with MATLAB (MathWorks, Natick, MA) based on edge detection and Bezier splines [37]. Three-dimensional region of interests (ROIs) of the full thickness of cartilage were generated and overlaid onto the reconstructed $T_{1 \rho}$ and $T_{2}$ maps. The ROI was defined to include the whole specimen through multiple slices in order to correlate MR measures with optical measures as detailed below. The mean, median, and standard deviation of $T_{1 \rho}$ and $T_{2}$ values were calculated after correcting ROIs to exclude fluid pixels.

\section{Optical Imaging}

The matrix metalloproteinase sensing probe (MMPSense 680, 13.3 $\mu \mathrm{M}$, VisEn Medical, Inc., Bedford, MA) was used to detect the MMP activity in all OA knee specimens. All specimens were incubated with MMPSense (diluted to $0.2 \mu \mathrm{M}$ in PBS) in the dark at $37^{\circ} \mathrm{C}$ for $2 \mathrm{~h}$. The resulting specimens were removed, washed with PBS, and subjected to optical reflectance imaging (IVIS 50, Caliper Life Sciences, Hopkinton, MA) using excitation and emission band-pass filters set at $640 \mathrm{~nm}( \pm 25)$ and $733 \mathrm{~nm}( \pm 37)$, respectively and a cooled CCD camera $\left(-90^{\circ} \mathrm{C}\right)$ to detect the fluorescence signal emitted by the MMPSense probe at $695 \mathrm{~nm}$. All images were collected using the same field of view with binning set at $4 \mathrm{~s}$, f-stop at $2 \mathrm{~s}$, and exposure time at $1 \mathrm{~s}$. All images were adjusted using the same threshold. The ROI was defined to include the entire specimen. The same criteria were used to define the ROI for all samples tested. Total photon efficiency, representing the radiance of the specimen normalized against the excitation intensity, was measured in the ROIs and subtracted from background. All optical measurements were tabulated with the $T_{1 \rho}$ and $T_{2}$ MR measurements. Analysis of variance (ANOVA) was used to evaluate the linear regression of the optical and MR imaging data.

\section{Histology}

Following ex vivo MR and optical imaging, 3-mm thick sagittal histological sections were obtained from the same plane as the sagittal ex vivo MR imaging. In addition, the exact location of the histology sections was recorded carefully using anatomical and landmark measurements. Locations to section were selected based on elevated MMP signals as determined by photon efficiency in optical imaging. Two sections were obtained for histological analysis from regions with high and low optical signals. These sections were fixed in $10 \%$ formalin and embedded in paraffin. Each section spanned the entire sagittal length of the specimen (approximately $4 \mathrm{~cm}$ ). The samples were stained with hematoxylin and eosin (H\&E) for morpho- 
logical measurements to give qualitative evaluation of cartilage degeneration.

\section{Results}

\section{$M R$ and Optical Imaging of OA Knee Specimens}

Quantitative 3-D $T_{1}$-weighted imaging of the OA knee specimens shows mean $T_{1 \rho}$ relaxation times ranging from 48 to $75 \mathrm{~ms}$. The corresponding color-coded $T_{1 \rho}$ maps overlaid on the SPGR images (Fig. 2) reveal regions with long relaxation times and hence severe loss of PGs [15]. In the same data set, $T_{2}$-weighted imaging shows long $T_{2}$ relaxation times (36-69 ms) in all samples (Table 1). 2-D optical fluorescence signals from MMPSense show a significant upregulation of MMP activities in all samples with total photon efficiency ranging from $3.76 \times 10^{-5}$ to $7.89 \times$ $10^{-4} \mathrm{~cm}^{2}$ (Fig. 3b; Table 1). No signal is apparent in the absence of MMPSense (Fig. 3a). The linear regression model of optical photon efficiency plotted against MR $T_{1 \rho}$ values shows a positive correlation (correlation coefficient, $\left.R^{2}=0.8936\right)$ with increasing optical signal corresponding to long $T_{1 \rho}$ relaxation time (Fig. 4 ). ANOVA test was applied to evaluate the regression model. The corresponding $P$ value was calculated at 0.0044 , indicating a significant relationship between the optical and $T_{1 \rho}$ MR measurements. There is no significant correlation between optical and $T_{2}$ or $T_{1 \rho}$ and $T_{2}$ measurements.

\section{Histological Analysis}

A femoral condyle specimen (K040MPFC) treated with MMPSense 680 was examined by optical and MR imaging. A high optical signal was observed in the medial compartment that directly coincides with the long $T_{1 \rho}$ relaxation captured by MR imaging (Fig. 5a, c), whereas the region of low optical signal corresponds with shorter $T_{1 \rho}$ relaxation time in the lateral compartment (Fig. 5b). Two sample slices were removed at regions with high and low optical signals for histological analysis. The corresponding H\&E staining shows that the region with high MMP optical signal has severe fissuring and clefting, disorganized tidemark as well as the loss of cellularity (Fig. 5d). On the contrary, the region with low MMP optical signal shows minimal clefting, normal tidemark, and normal cellularity (Fig. 5e).

\section{Discussion}

From the onset to the progression of OA is a result of multiple cascading molecular events that lead to the breakdown of macromolecules and ultimately the loss of the overall structural integrity of the cartilage [38]. Investigations of early molecular events and macromolecular changes in OA have helped us to gain a better understanding of the disease process from cells [22] to animals [39] and humans $[35,40]$. Although these studies are extremely valuable, there is little evidence of these molecular and macromolecular markers working synergistically towards OA development. For instance, $T_{2}$ relaxation, which measures the increasing water mobility in damaged collagen, provides a glimpse of a degenerative event in an already damaged collagen network; it reveals no information about the early proteolytic process that is responsible for collagen degradation. Of equal importance is the realization that although $T_{1 \rho}$ quantification has been shown to correlate with the loss of PG macromolecules, such measurements report a disease process that has already taken place, and earlier biochemical events that are responsible for the digestion of PG still remain elusive. The ability to interrogate both molecular and macromolecular changes in the disease process, therefore, will not only provide us with a more comprehensive profile of the degradation mechanism in OA, it may also open new

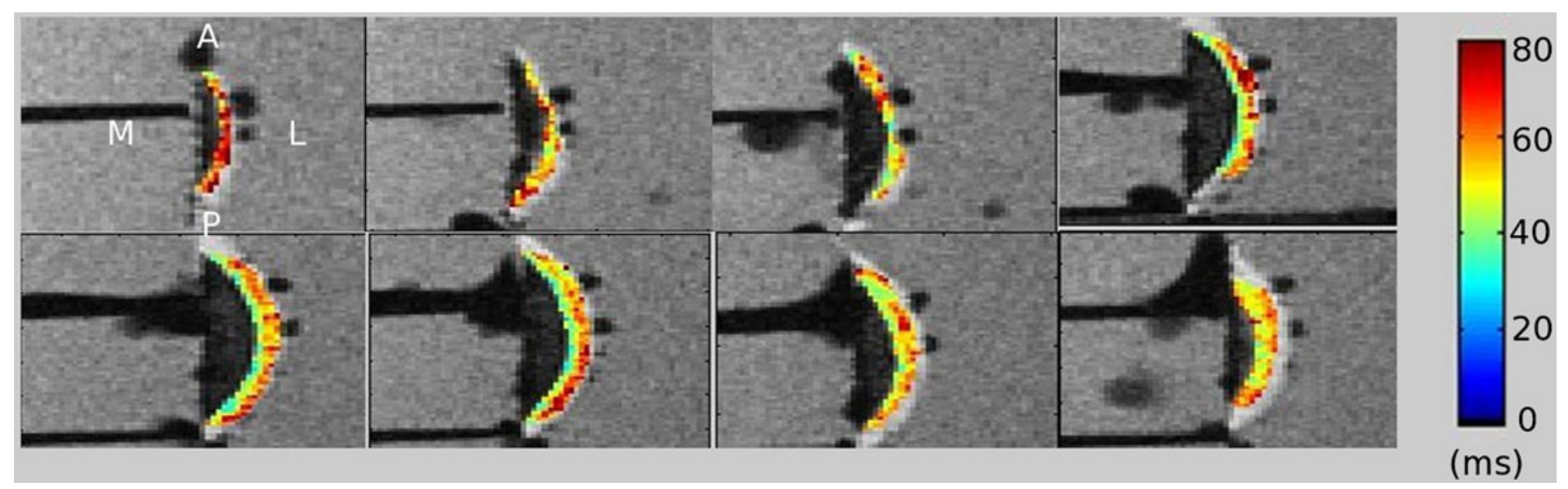

Fig. 2. A representative image of $T_{1 \rho}$ mapping of a human femoral condyle specimen. All specimens were imaged in their respective physiological positions to simulate the in vivo orientation and position: $M$ medial, $L$ lateral, $A$ anterior, and $P$ posterior. Images were scanned from medial to lateral positions. Cartilage $T_{1 \rho}$ relaxation times (ms) are overlaid with colorcoded SPGR images for visualization. 
Table 1. A summary of results from $T_{1 \rho}$ relaxation time, $T_{2}$ relaxation time, and optical measurements of human femoral condyles from patients diagnosed with severe OA

\begin{tabular}{lccc}
\hline Specimen & Average $3 \mathrm{D}-T_{1 \rho}(\mathrm{ms})$ & Average $T_{2}(\mathrm{~ms})$ & Photon efficiency $\left(\mathrm{cm}^{2}\right)$ \\
\hline K040 MPFC & $56.16 \pm 12.94$ & $40.85 \pm 8.65$ & $2.16 \mathrm{E}-04$ \\
K045 LPFC & $48.02 \pm 15.21$ & $36.22 \pm 7.53$ & $3.76 \mathrm{E}-05$ \\
K045 MPFC & $58.03 \pm 11.02$ & $51.94 \pm 22.55$ & $2.13 \mathrm{E}-04$ \\
K050 MPFC & $62.93 \pm 19.28$ & $43.61 \pm 13.33$ & $2.71 \mathrm{E}-04$ \\
K058 LPFC & $62.17 \pm 11.28$ & $37.87 \pm 10.64$ & $4.89 \mathrm{E}-04$ \\
K060 LPFC & $74.75 \pm 21.88$ & $47.34 \pm 12.55$ & $7.89 \mathrm{E}-04$ \\
\hline
\end{tabular}

avenues for therapeutic targets designed to halt the degenerative process at its earliest stages.

\section{Imaging Approach with Optical and MR}

Active MMP-9 and -13 are key enzymes that are responsible for the digestion of collagens and degradation of PGs during the early stages of OA. Upregulation of MMPs, therefore, could be an early sign of OA development. In fact, comprehensive gene profiling using real-time polymerase chain reaction in both animal [41] and human [42, 43] OA cartilages have revealed the overexpression of MMP-9 and 13. In particular, studies using canine elbow bone and cartilage have shown that the upregulation of MMP-9 and 13, correlate with radiographic evidence of the disease [41], suggesting expression of these two proteases may act as a predictor for OA severity.

The interrogation of proteolytic activities requires a detection method with high specificity and sensitivity. Although the peptide sequence on the MMPSense is sensitive toward a number of MMPs, it is predominantly cleaved by MMP-9 and -13, making this agent well suited for probing proteolytic activities in OA. The built-in "onoff" switch in MMPSense also allows it to have maximum

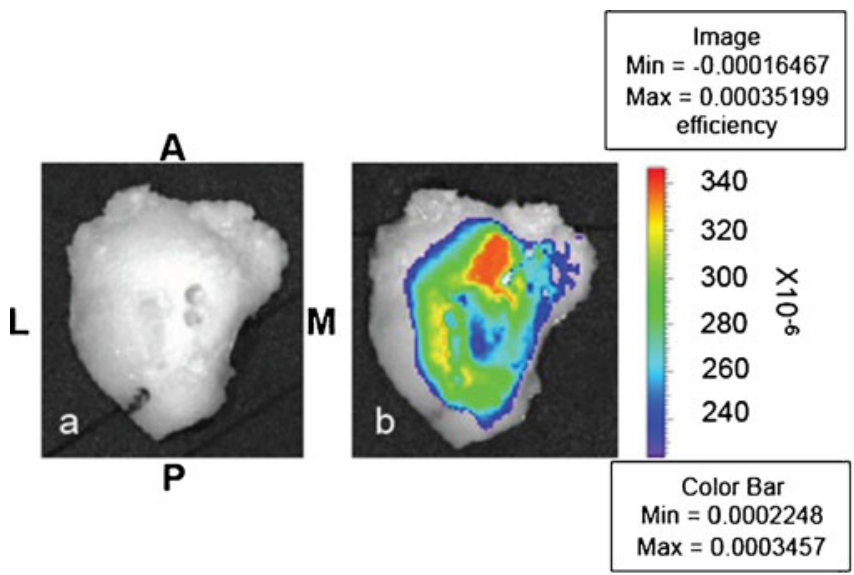

Fig. 3. A representative optical image of a human OA knee specimen. The orientation and positioning of the specimen is as follows: $M$ medial, $L$ lateral, $A$ anterior, and $P$ posterior. a Before incubation with MMPSense 680 with no detectable background signal; b after incubation with $0.2 \mu \mathrm{M}$ of MMPSense 680 in PBS for $2 \mathrm{~h}$. The intense optical signal signifies the upregulation of MMP activities. signal amplification, providing high detection sensitivity down to picomole concentration [44]. Importantly, because the optical signal is acquired in the near-IR region, interference from auto-fluorescence from biological tissues (such as cartilage and bone) is negligible [45]. The "turned on" MMP probe, therefore, is a direct measure of the MMP activity level without any interference from background signals.

$T_{1 \rho}$ quantification has shown great promise as a clinical tool to characterize cartilage degeneration that cannot be detected by standard MR imaging techniques. The $T_{1 \rho}$ relaxation represents the slow-motion interactions between motion-restricted water molecules and their macromolecular environment. Probing for these water molecule signals with $T_{1 \rho}$ reflects macromolecular changes such as PG loss. Previous studies have reported that $T_{1 \rho}$ relaxation rate $\left(1 / T_{1 \rho}\right)$ correlates with PG concentration in cartilage [8]. Studies in specimens and in patients have shown collective evidence of elevated $T_{1 \rho}$ in OA cartilage [10, $15,35,46-48]$. The $T_{2}$ value in cartilage is known to be affected by the overall water mobility, collagen content, and structural features within the ROI. Although it is a rather less sensitive measurement, elevated $T_{2}$ values have been observed in OA $[13,49]$. In this study, both $T_{1 \rho}$ and $T_{2}$ values of the OA knee specimens are relatively high ranging from 48 to $75 \mathrm{~ms}$ and from 36 to $69 \mathrm{~ms}$, respectively, suggesting the well-recognized heterogeneity of cartilage degeneration [50].

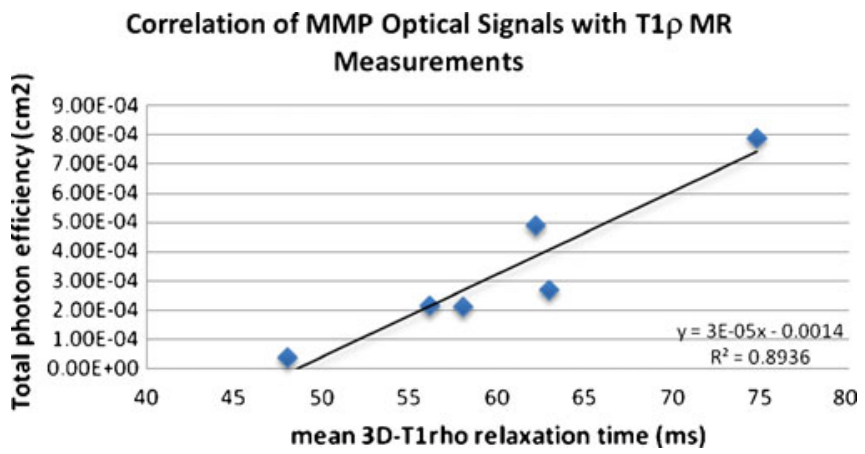

Fig. 4. The MMP optical signal was plotted against the MR $T_{1 \rho}$ measurement. Six different samples were used. A linear regression model showing an increase of MMP optical signal with $T_{1 \rho}$ measurement with $R^{2}$ at 0.8936 . The corresponding $P$ value was calculated at 0.0044 , indicating a significant relationship of the optical and MR measurements. 

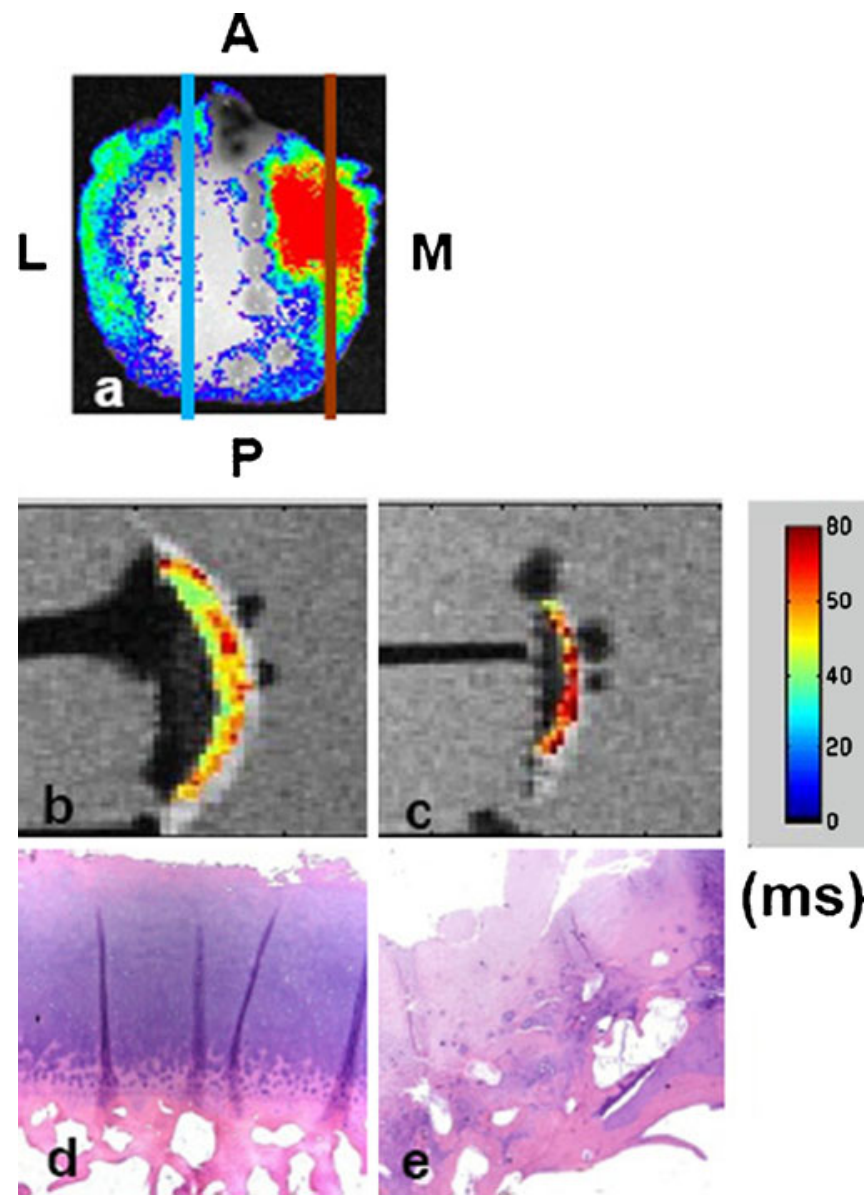

(ms)

Fig. 5. A femoral condyle (K040MPFC) was treated with MMPSense 680 and examined by optical and MR imaging. The optical signal was normalized against the background excitation and expressed in photon efficiency. The specimen was imaged in its respective physiological positions to simulate the in vivo orientation and position: $M$ medial, $L$ lateral, $A$ anterior, and $P$ posterior. Signals observed here indicate high MMP activities in the medial compartment (a). Two slices were removed from high (red) and low (b/ue) optical regions for histological analysis. b, c Color-coded $T_{1 \rho}$ maps overlaid on a 3-Tesla MR image with (b) corresponding to the low optical signal region and (c) corresponding to the high optical signal region. The $T_{1 \rho}$ relaxation value is ranging from 40 to $60 \mathrm{~ms}$ with the highest value coded as red. The intense $T_{1 \rho}$ region signifies the severe loss of proteoglycans. d, e The hematoxylin and eosin staining showing the region with low MMP optical signal (d) has minimal clefting, normal tidemark, and normal cellularity. On the contrary, the region with high MMP optical signal exhibits severe fissuring and clefting, disorganized tidemark as well as the loss of cellularity.

\section{MMP and Proteoglycans}

When comparing the optical and MR measurements, we recognized that the optical reflectance measurement is limited to providing the overall surface signal from the specimen without any depth information. During MR image analysis, instead of measuring each layer of the cartilage matrix, we opted to measure $T_{1 \rho}$ and $T_{2}$ for the full thickness of the cartilage to allow for comparable imaging data from MR and optical.

MMP's involvement in OA degeneration can be realized by examining both optical and MR data. From the linear regression model with MMP activities expressed in normalized photon efficiency plotted against the amount of PG loss measured by $T_{1 \rho}$, there is a positive correlation between the two $\left(R^{2}=0.8936, P\right.$ value of 0.0044$)$. Although the present study is limited by the lack of direct PG content quantification measured by conventional biochemical assays, a recent study from our group using human osteoarthritic cartilage (specimens similar to that used in this study) showed a significant correlation between PG contents and $T_{1 \rho}$ quantification [11]. Collectively, the relationship found between the optical and MR $T_{1 \rho}$ data not only confirms the presence of active MMPs at the late stage of OA, it also implicates MMPs' involvement in PG degradation. Although $T_{2}$ measurements give the gross water content of the knee cartilage, and there is no observable correlation with $T_{1 \rho}$ or the optical data, the high water content suggests that the collagen matrix is compromised and is contributing to the overall structural instability and severity of OA in patients.

\section{Histological Findings}

The histological result is best visualized with both optical and MR data (Fig. 5a-c). Two slices taken from an OA knee specimen with regions of both high and low MMP activity (as indicated from the optical imaging) were subjected to H\&E staining. The sample taken from the high MMP region not only exhibits high $T_{1 \rho}$ relaxation, it also displays an abnormal structure and cellularity by histology, which are the classic signs of OA. The sample taken from the low MMP optical region shows a much less intense $T_{1 \rho}$ map, minimal clefting, normal tidemark, and cellularity. Because cartilage consists of only one cell type [51], chondrocytes, H\&E staining did not reveal any inflammation cell distribution. Nonetheless, our collective findings suggest a possible link between increasing MMPs activity and more severe PG loss and cartilage abnormalities.

\section{Conclusions}

In this study, we have demonstrated the utility of optical imaging with MMPSense to characterize the upregulation of MMPs in human OA cartilage. Based on the MR $T_{1 \rho}$ and $T_{2}$ measurements, all these specimens have severe PG loss and high water mobility in the damaged cartilage. The histology data further confirms the abnormality of the cartilage with high optical MMP signal and intense $T_{1 \rho}$ mapping. The relationship between the MMP optical data and the $T_{1 \rho}$ measurements also implicates MMP's involvement in the OA disease process that ultimately leads to macromolecular 
PG degradation. Further investigations combining optical and MR imaging of OA may yield useful molecular and macromolecular information of the disease pathway, offering insights to new tool development for OA early detection, treatment, and/or prevention.

Acknowledgements. This work was supported in part by the National Institute of Health (5 RO1AG07762 and 5 RO1AR046905). The authors would like to thank Dr. Andrew Horvai for the assistance with histology and Dr. Jeffery Peterson (Perkin Elmer) for his helpful discussions.

Conflict of Interest. The authors declare that they have no conflict of interest.

Open Access. This article is distributed under the terms of the Creative Commons Attribution Noncommercial License which permits any noncommercial use, distribution, and reproduction in any medium, provided the original author(s) and source are credited.

\section{References}

1. AHRQ (2008) Big Money: Cost of 10 Most Expensive Health Conditions Near $\$ 500$ Billion. AHRQ News and Numbers, January 23, 2008. http://www.ahrq.gov/news/nn/nn012308.htm. Accessed 13 Jan 2010

2. Srikulmontree T (2006) Osteoarthritis: The American College of Rheumatology. Downloaded from http://www.rheumatology.org/practice/clinical/patients/diseases and conditions/osteoarthritis.asp. Accessed 13 Jan 2010

3. Link T, Stahl R, Woertler K (2007) Cartilage imaging: motivation, techniques, current and future significance. Eur Radiol 17:1135-1146

4. Burstein D, Gray M, Mosher T, Dardzinski B (2009) Measures of molecular composition and structure in osteoarthritis. Radiol Clin North Am 47:675-686

5. Borthakur A, Mellon E, Niyogi S, Witschey W, Kneeland JB, Reddy R (2006) Sodium and T1rho MRI for molecular and diagnostic imaging of articular cartilage. NMR Biomed 19:781-821

6. Nieminen MT, Rieppo J, Toyras J et al (2001) T2 relaxation reveals spatial collagen architecture in articular cartilage: a comparative quantitative MRI and polarized light microscopic study. Magn Reson Med 46:487-493

7. Watrin A, Ruaud JPB, Olivier PTA et al (2001) T2 mapping of rat patellar cartilage. Radiology 219:395-402

8. Akella SV, Regatte RR, Gougoutas AJ et al (2001) Proteoglycaninduced changes in T1rho-relaxation of articular cartilage at $4 \mathrm{~T}$. Magn Reson Med 46:419-423

9. Duvvuri U, Kudchodkar S, Reddy R, Leigh JS (2002) T(1rho) relaxation can assess longitudinal proteoglycan loss from articular cartilage in vitro. Osteoarthritis Cartilage 10:838-844

10. Wheaton AJ, Dodge GR, Elliott DM, Nicoll SB, Reddy R (2005) Quantification of cartilage biomechanical and biochemical properties via T1rho magnetic resonance imaging. Magn Reson Med 54:1087-1093

11. Li X, Cheng J, Lin K et al (2010) Quantitative MRI using T(1rho) and T (2) in human osteoarthritic cartilage specimens: correlation with biochemical measurements and histology. Magn Reson Imaging (in press)

12. Mosher TJ, Dardzinski BJ, Smith MB (2000) Human articular cartilage: influence of aging and early symptomatic degeneration on the spatial variation of T2-preliminary findings at $3 \mathrm{~T}$. Radiology 214:259-266

13. Dunn TC, Lu Y, Jin H, Ries MD, Majumdar S (2004) T2 relaxation time of cartilage at MR imaging: comparison with severity of knee osteoarthritis. Radiology 232:592-598

14. Regatte RR, Akella SV, Borthakur A et al (2003) In vivo proton MR three-dimensional T1rho mapping of human articular cartilage: initial experience. Radiology 229:269-274

15. Li X, Benjamin Ma C, Link TM et al (2007) In vivo $\mathrm{T}(1$ rho) and $\mathrm{T}(2)$ mapping of articular cartilage in osteoarthritis of the knee using $3 \mathrm{~T}$ MRI. Osteoarthritis Cartilage 15:789-797

16. Bonnet CS, Walsh DA (2005) Osteoarthritis, angiogenesis and inflammation. Rheumatology 44:7-16
17. Martel-Pelletier J, Welsch DJ, Pelletier JP (2001) Metalloproteases and inhibitors in arthritic diseases. Best Pract Res Clin Rheumatol 15:805829

18. Aigner T, Fundel K, Saas J et al (2006) Large-scale gene expression profiling reveals major pathogenetic pathways of cartilage degeneration in osteoarthritis. Arthritis Rheum 54:3533-3544

19. Murphy G, Knauper V, Atkinson S et al (2002) Matrix metalloproteinases in arthritic disease. Arthritis Res 4(Suppl 3):S39-S49

20. Sternlicht MD, Werb Z (2001) How matrix metalloproteinases regulate cell behavior. Annu Rev Cell Dev Biol 17:463-516

21. Van Wart HE, Birkedal-Hansen H (1990) The cysteine switch: a principle of regulation of metalloproteinase activity with potential applicability to the entire matrix metalloproteinase gene family. Proc Natl Acad Sci USA 87:5578-5582

22. Dreier R, Grässel S, Fuchs S, Schaumburger J, Bruckner P (2004) ProMMP-9 is a specific macrophage product and is activated by osteoarthritic chondrocytes via MMP-3 or a MT1-MMP/MMP-13 cascade. Exp Cell Res 297:303-312

23. Weissleder R, Mahmood U (2001) Molecular imaging. Radiology 219:316-333

24. Kopka K, Breyholz HJ, Wagner S et al (2004) Synthesis and preliminary biological evaluation of new radioiodinated MMP inhibitors for imaging MMP activity in vivo. Nucl Med Biol $31: 257-267$

25. Jaffer FA, Weissleder R (2004) Seeing within: molecular imaging of the cardiovascular system. Circ Res 94:433-445

26. Mahmood U, Weissleder R (2003) Near-infrared optical imaging of proteases in cancer. Mol Cancer Ther 2:489-496

27. Bremer C, Bredow S, Mahmood U, Weissleder R, Tung C-H (2001) Optical imaging of matrix metalloproteinase-2 activity in tumors: feasibility study in a mouse model. Radiology 221:523-529

28. Seltzer JL, Akers KT, Weingarten H, Grant GA, McCourt DW, Eisen AZ (1990) Cleavage specificity of human skin type IV collagenase (gelatinase). Identification of cleavage sites in type I gelatin, with confirmation using synthetic peptides. J Biol Chem 265:20409-20413

29. Peterson J, Kossodo S, Korideck H et al (2008) Optical imaging of MMPSense as a biomarker of matrix metalloprotease activity in vivo. In: Abstract no. 1130 of the 99th AACR Annual Meeting. San Diego, CA

30. Groves K, Kossodo S, Handy E et al (2009) Abstract \#LB-68: In vivo imaging of treatment effects using a novel near infrared labeled agent. AACR Meeting Abstracts: LB-68, 2009

31. Gabriely G, Wurdinger T, Kesari S et al (2008) MicroRNA 21 promotes glioma invasion by targeting matrix metalloproteinase regulators. Mol Cell Biol 28:5369-5380

32. Littlepage LE, Sternlicht MD, Rougier N et al (2010) Matrix metalloproteinases contribute distinct roles in neuroendocrine prostate carcinogenesis, metastasis, and angiogenesis progression. Cancer Res 70:2224-2234

33. Schaffer BS, Grayson MH, Wortham JM et al (2010) Immune competency of a hairless mouse strain for improved preclinical studies in genetically engineered mice. Mol Cancer Ther 9:2354-2364

34. Peterson J, LaBranche T, Vasquez K et al (2010) Optical tomographic imaging discriminates between disease-modifying anti-rheumatic drug (DMARD) and non-DMARD efficacy in collagen antibody-induced arthritis. Arthritis Res Ther 12:R105

35. Li X, Han ET, Busse RF, Majumdar S (2008) In vivo T(1rho) mapping in cartilage using 3D magnetization-prepared angle-modulated partitioned k-space spoiled gradient echo snapshots (3D MAPSS). Magn Reson Med 59:298-307

36. Pai A, Li X, Majumdar S (2008) A comparative study at $3 \mathrm{~T}$ of sequence dependence of $\mathrm{T} 2$ quantitation in the knee. Magn Reson Imaging 26:1215-1220

37. Carballido-Gamio J, Krug R, Huber MB et al (2009) Geodesic topological analysis of trabecular bone microarchitecture from highspatial resolution magnetic resonance images. Magn Reson Med $61: 448-456$

38. Goldring MB, Goldring SR (2010) Articular cartilage and subchondral bone in the pathogenesis of osteoarthritis. Ann N Y Acad Sci 1192:230-237

39. Kamekura S, Hoshi K, Shimoaka $\mathrm{T}$ et al (2005) Osteoarthritis development in novel experimental mouse models induced by knee joint instability. Osteoarthritis Cartilage 13:632-641 
40. David-Vaudey E, Ghosh S, Ries M, Majumdar S (2004) T2 relaxation time measurements in osteoarthritis. Magn Reson Imaging 22:673-682

41. Clements DN, Fitzpatrick N, Carter SD, Day PJ (2009) Cartilage gene expression correlates with radiographic severity of canine elbow osteoarthritis. Vet J 179:211-218

42. Kevorkian L, Young DA, Darrah C et al (2004) Expression profiling of metalloproteinases and their inhibitors in cartilage. Arthritis Rheum 50:131-141

43. Davidson RK, Waters JG, Kevorkian L et al (2006) Expression profiling of metalloproteinases and their inhibitors in synovium and cartilage. Arthritis Res Ther 8:R124

44. Graves EE, Ripoll J, Weissleder R, Ntziachristos V (2003) A submillimeter resolution fluorescence molecular imaging system for small animal imaging. Med Phys 30:901-911

45. Weissleder R, Tung CH, Mahmood U, Bogdanov A Jr (1999) In vivo imaging of tumors with protease-activated near-infrared fluorescent probes. Nat Biotechnol 17:375-378
46. Li X, Pai A, Blumenkrantz G et al (2009) Spatial distribution and relationship of T1rho and T2 relaxation times in knee cartilage with osteoarthritis. Magn Reson Med 61:1310-1318

47. Regatte RR, Akella SV, Lonner JH, Kneeland JB, Reddy R (2006) T1rho relaxation mapping in human osteoarthritis (OA) cartilage: comparison of T1rho with T2. J Magn Reson Imaging 23:547-553

48. Stahl R, Luke A, Li X et al (2009) T1rho, T2 and focal knee cartilage abnormalities in physically active and sedentary healthy subjects versus early OA patients-a 3.0-Tesla MRI study. Eur Radiol 19:132-143

49. Mosher TJ, Dardzinski BJ (2004) Cartilage MRI T2 relaxation time mapping: overview and applications. Semin Musculoskelet Radiol $8: 355-368$

50. Brandt KD, Doherty M, Lohmander LS (eds) (1998) Osteoarthritis. Oxford University Press, Inc., New York

51. Watanabe HY, Yoshihiko, Kimata (1998) Koji Roles of Aggrecan, a large chondroitin sulfate proteoglycan, in cartilage structure and function. J Biochem 124:687 